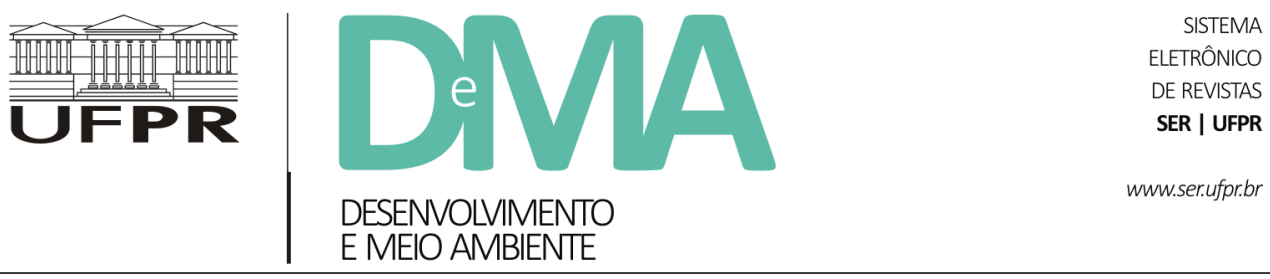

\title{
Impacto da implantação do BRT na arborização da região central de Palmas, Tocantins
}

\section{The impact of the implementation of Bus Rapid Transit (BRT) on the trees of the central region of Palmas, Tocantins}

\author{
Renato Torres PINHEIRO ${ }^{1 *}$, Dianes Gomes MARCELINO², Dieyson Rodrigues de MOURA ${ }^{2}$ \\ ${ }^{1}$ Universidade Federal do Tocantins (UFT), Palmas, TO, Brasil. \\ ${ }^{2}$ Ecótono Engenharia, Palmas, TO, Brasil \\ *E-mail de contato: renaxas@hotmail.com
}

Artigo recebido em 24 de outubro de 2017, versão final aceita em 19 de junho de 2018.

RESUMO: $\quad$ O crescimento acelerado e desordenado das cidades contribui para o aumento da degradação ambiental desencandeada principalmente pela falta de infraestrutura básica, fragmentação e perda de habitat. Todos estes fatores culminam na perda de biodiversidade e diminuição da qualidade de vida dos munícipes. Neste contexto, a manutenção de áreas verdes nos centros urbanos torna-se indispensável para minimizar os efeitos da urbanização sobre o meio ambiente e a população urbana, uma vez que desempenham diversos serviços ambientais, tais como paisagísticos, ecológicos, climáticos, recreativos e psicológicos. Desta forma, avaliou-se neste trabalho o impacto da implantação do sistema de transporte coletivo BRT sobre a arborização da parte central do canteiro da Avenida Teotônio Segurado em Palmas, Tocantins, discutindo seus efeitos sobre a estrutura ecológica urbana e os serviços ambientais. Analisamos ainda uma proposta alternativa de traçado da canaleta de circulação do BRT visando a reduzir o seu impacto sobre a arborização local. Foram inventariados 3.980 indivíduos arbóreos de 112 espécies nessa avenida, totalizado 72,64 ind./ ha. O impacto sobre a arborização do projeto original da canaleta de circulação do BRT para a área central da via é cerca de $30 \%$ maior do que o traçado alternativo proposto neste trabalho, sendo suprimidos pelo menos 456 indivíduos arbóreos. Em termos de composição e importância biológica e ecológica, o traçado do projeto original ocasionará a supressão de 23 espécies nativas do cerrado a mais quando comparado ao traçado alternativo. Os impactos sobre a arborização do projeto original das canaletas de circulação do BRT implicam na perda de diversos serviços ambientais proporcionado pela vegetação da Av. Teotônio Segurado, dentre os quais a amenização da poluição do ar e sonora, a diminuição das ilhas de calor, a melhoria da qualidade de vida e a manutenção e dispersão de espécies, sendo o traçado alternativo proposto a opção ambiental e socioeconomicamente mais viável.

Palavras-chave: impacto ambiental; sistema BRT; cidades sustentáveis; áreas verdes urbanas; mobilidade urbana. 
ABSTRACT: The accelerated and disordered growth of cities contributes to the increase in environmental deterioration unleashed mainly by the lack of basic infrastructure, fragmentation and loss of habitats. All these factors culminate in the loss of biodiversity and decrease of the quality of life of the citizens. In this context, the maintenance of green areas in urban centers becomes indispensable to minimize the effects of urbanization on the environment and the urban population, since they perform various environmental services such as landscape, ecological, climatic, recreational and psychological. In this manner, through this project, we evaluated the impact of the implementation of the BRT public transportation system on the afforestation of the central plat of the Teotônio Segurado Avenue in Palmas, Tocantins, discussing its effects on urban ecological structure and environmental services. We also analyzed an alternative proposal for tracing the BRT circulation channel in order to reduce its impact on local afforestation. A total of 3,980 arboreal individuals of 112 species were registered on the Teotônio Segurado Avenue, totaling 72.64 ind./ha. The impact on the afforestation of the original BRT circulation channel on the central area of the avenue is about $30 \%$ greater than the alternative route proposed in this study, with at least 456 individuals being suppressed. In terms of composition and biological and ecological importance, the design of the original project will cause the suppression of 23 more native species of the cerrado when compared to the alternative route. The impacts on the afforestation of the original design of the BRT circulation channels imply the loss of several environmental services provided by the vegetation of the Teotônio Segurado Avenue, such as mitigation of air pollution and noise, decrease of the islands of heat, improvement of the quality of life, maintenance and dispersion of species, therefore, revealing that the proposed alternative route is the most viable environmental and socioeconomical option.

Keywords: environmental impact; BRT System; sustainable cities; urban green areas; urban mobility.

\section{Introdução}

A urbanização, além de causar uma série de impactos nos ambientes naturais, é considerada uma das principais fontes de destruição e degradação ambiental em nível global. O crescimento urbano, que ocorre na maioria dos casos de forma desordenada, além de ter impactos sobre o meio ambiente, gera inúmeros problemas físico-estruturais, sociais e econômicos (Hogan et al., 2001; Chaves, 2011).

A partir da década de 1980, novos conceitos como o da sustentabilidade e a mobilização global, em torno das questões ambientais e ecológicas, também repercutiram acerca da importância do meio ambiente urbano (Alves, 2009). Mais recentemente, o verde urbano tem sido pensado e planejado como parte da rede estruturante urbana, formando uma estrutura ecológica que integra organicamente o verde da cidade com o do entorno, englobando os componentes ambientais, sociais e econômicos, de forma a promover e prover múltiplos bens e serviços ambientais para a população (Ferreira, 2010).

A estrutura ecológica, por sua vez, deverá ser compreendida como mais um elemento da infraestrutura urbana, assim como a rede de abastecimento de água, energia elétrica, estradas e outras (Ferreira et al., 2004). Porém, segundo o Forest Research (2010), estes benefícios serão alcançados com maior sucesso se o verde urbano estiver integrado com os espaços construídos. E mais: alguns bens e serviços ambientais dependem da distribuição e de uma forte conectividade da infraestrutura verde urbana. A junção dos elementos que compõem a estrutura ecológica urbana é feita por uma rede de corredores verdes que ligam grandes áreas verdes não lineares ou manchas de vegetação, integrando áreas de grande valor ecológico, cultural e paisa- 
gístico, compatibilizadas com a atividade humana (Ferreira et al., 2004; Benedict \& McMahon, 2006; Quintas \& Curado, 2009).

À medida que as cidades crescem, as necessidades da população aumentam, e a mobilidade urbana passa a ser uma demanda cada vez mais presente e compatível com os princípios da sustentabilidade. No contexto socioeconômico urbano, a mobilidade sustentável é composta por ações sobre o uso e ocupação do solo e sobre a gestão dos transportes, buscando melhorar a qualidade de vida da população no presente e no futuro. Na conjuntura ambiental, alia-se a novas tecnologias para redução do impacto ambiental associada a fatores de redução no consumo de energia, da poluição do ar e sonora, e ampliação do conforto urbano por meio da instalação de calçadas, ciclovias, segurança em travessias e arborização viária (Campos, 2013).

Conciliar a urbanização com a preservação dos recursos naturais e o desenvolvimento sustentável é uma tarefa complexa, devendo cada caso ser avaliado individualmente. A cidade de Palmas, Tocantins, foi a última capital brasileira projetada do século XX (Carvalhedo \& Lira, 2009). Concebida dentro de uma perspectiva humanístico-ecológica, apresenta-se hoje como a capital brasileira com maior taxa de crescimento geométrico (IBGE, 2017): um cenário que pede ações de planejamento eficientes e sustentáveis.

Um dos eixos estratégicos de desenvolvimento que prevê o uso do território de forma mais equilibrada, em Palmas, envolve o transporte coletivo, tendo o sistema Bus Rapid Transit (BRT) como modelo escolhido. Porém, apesar de ser um modelo classificado como sustentável, frente a outros modais, amplamente difundido e exitoso em diversas cidades do mundo (Branco, 2013), a sua implanta- ção em Palmas está prevista para ocorrer no canteiro central da avenida Teotônio Segurado. Trata-se do principal corredor verde urbano da cidade, com mais de $20 \mathrm{Km}$ de extensão, responsável pela conexão das regiões norte e sul, com elevada densidade e riqueza de árvores (mais de 110 espécies), e trechos onde a vegetação nativa do Cerrado é abundante e bem preservada, além de interligar a principais áreas verdes e unidades de conservação urbanas do município (Palmas, 2015).

Os canteiros centrais da avenida Teotônio Segurado variam quanto ao tamanho, desenho paisagístico e composição de espécies. Basicamente existem três tipos de canteiros, aqueles com intervenção paisagística, canteiros sem tratamento paisagístico onde a vegetação foi parcialmente ou totalmente suprimida e canteiros sem paisagismo com predomínio de espécies nativas autóctones.

No presente estudo, avaliamos as consequências da implantação do sistema de transporte coletivo BRT sobre a arborização do canteiro da avenida Teotônio Segurado, discutindo seus efeitos sobre a estrutura ecológica urbana e os serviços ambientais. Analisamos ainda uma proposta alternativa de traçado da canaleta de circulação do BRT procurando reduzir o seu impacto sobre a arborização local.

\section{Materiais e Métodos}

\section{1. Área de estudo}

A cidade de Palmas está situada na região central do Estado do Tocantins e localizada no paralelo $10^{\circ} 11^{\prime} 04^{\prime \prime}$ Sul e do meridiano $48^{\circ} 20^{\prime} 01^{\prime \prime}$ Oeste. Fundada em maio de 1989, possui atualmente uma 
população estimada de 286.787 habitantes (IBGE, 2017). O clima na região é quente e caracterizado por duas estações bem definidas: uma chuvosa, entre os meses de outubro a abril; e outra seca, entre os meses de maio a setembro. O índice pluviométrico varia de 1.500 a $1.900 \mathrm{~mm} /$ ano. A temperatura média anual é de $26^{\circ} \mathrm{C}$, sendo setembro o mês mais quente, com média máxima de $36^{\circ} \mathrm{C}$ e julho o mais frio, com média mínima de $15,5^{\circ} \mathrm{C}$. A vegetação natural é típica do cerrado, havendo fisionomias

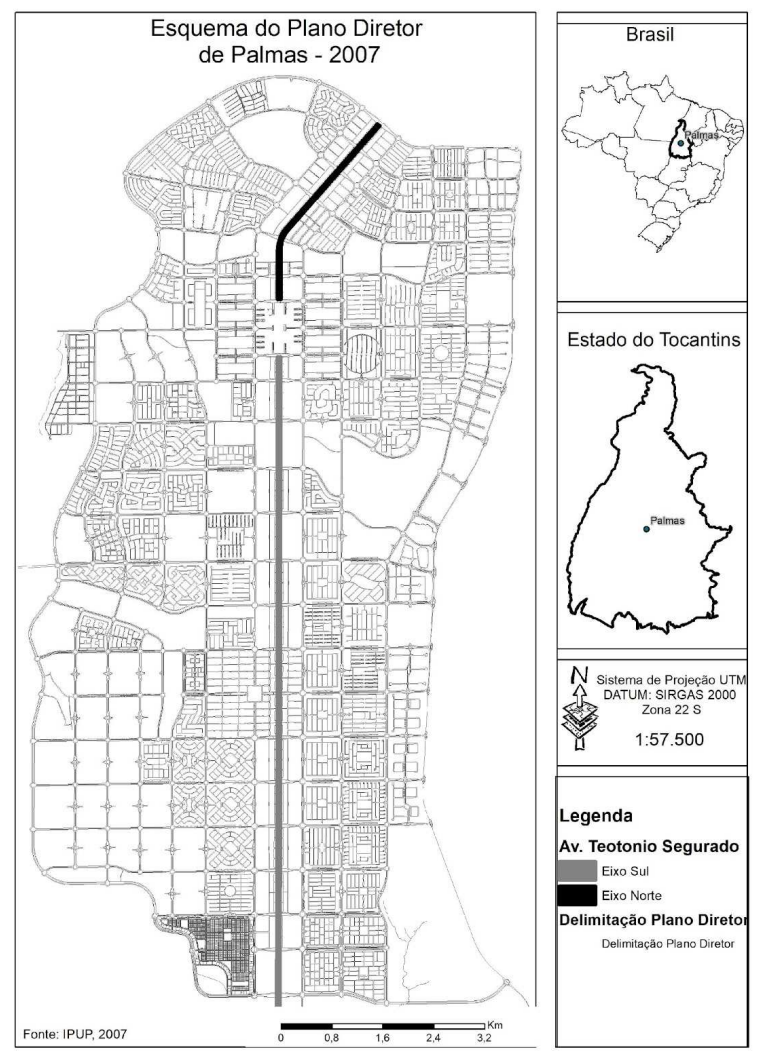

FIGURA 1 - Esquema do Plano Diretor de Palmas, delimitando os eixos sul e norte da Avenida Teotônio Segurado, Palmas, Tocantins. FONTE: Instituto de Planejamento Urbano de Palmas (2007). Adaptado por Dieyson Moura. florestais (cerradão e mata de galeria), campestres (campo sujo), e savânicas (cerrado sensu stricto) que formam a paisagem local (Tocantins, 2012).

O estudo foi realizado na Avenida Teotônio Segurado, principal via arterial da cidade, responsável pela conexão das regiões norte e sul, com uma extensão de aproximadamente $20 \mathrm{~km}$. Ela possui um canteiro central 45 metros de largura, que se estende por toda a área planejada da cidade, sendo cortado por diversas avenidas transversais no sentido Leste/Oeste que a dividem em 22 canteiros, cujas dimensões variam de 200x45m a 790x45m, podendo variar também em função do relevo e da presença de dois ribeirões e do ordenamento urbano (Figura 1).

\subsection{Metodologia}

A coleta de dados foi realizada em campo pelos autores, mediante o preenchimento de planilha eletrônica digital incorporada em um coletor de dados Mesa Juniper System ${ }^{\circledR}$. Foram coletados dados de todos os indivíduos arbóreos, com informações relativas à espécie (nome científico e popular) e origem, sendo as espécies classificadas em: (N) nativas do cerrado, espécies com ocorrência comprovada no bioma cerrado; (EC) exótica cerrado, espécies nativas brasileiras, mas com ocorrência fora do bioma cerrado e (EB) exótica Brasil, espécies originárias de outros países. Para cada categoria foram obtidos dados referentes à riqueza de espécies e densidade (indivíduos arbóreos por hectare - ind./ha), dados sobre altura, porte, estado de desenvolvimento (referentes a impermeabilização e condição do solo) e fitossanidade. Técnicas analíticas descritivas foram utilizadas para organizar os dados e investigá-los. 
Com o objetivo de identificar as transformações advindas da implantação do BRT no canteiro central da Av. Teotônio Segurado, calculou-se o impacto sobre sua arborização considerando dois traçados da canaleta de circulação. Um seguindo o desenho original, projetado para a parte central do canteiro com 15 metros; e outro, alternativo com duas canaletas de 7,5 metros em cada uma das extremidades do canteiro, compondo uma faixa adicional de rolamento à avenida. Estas dimensões são conservadoras e não levam em consideração que a obras de implantação deverão gerar impactos além dos 15 metros predefinidos e também não consideram a implantação das estações que ocuparão toda a extensão do canteiro central.

As espécies vegetais foram identificadas em campo. No caso de dúvidas foram coletadas amostras encaminhadas para identificação por especialistas no Herbário da Universidade Federal do Tocantins, Campus de Porto Nacional. Informações taxonômicas das espécies brasileiras foram obtidas na base de dados do Jardim Botânico do Rio de Janeiro (Flora do Brasil, 2017) e Lorenzi (2002; 2013; 2016), e das espécies estrangeiras em The Plant List (2013).

\section{Resultados}

A Av. Teotônio Segurado possui 22 canteiros que variam entre 0,9 e 3,5 ha, ocupando uma área de aproximadamente 54,7 ha $(12.160 \times 45$ metros), onde foram inventariados 3.980 indivíduos arbóreos de 112 espécies, totalizando 72,6 indiv/ ha. As espécies mais abundantes foram o Pequi Caryocar brasiliense Cambess. com 15\% do total de árvores amostradas, seguida pelo Canudo-de-pito
Mabea fistulifera Mart. com 7,4\% e a Cachamorra Tachigali rubiginosa (Mart. ex Tul.) Oliveira-Filho com 7,1\%, (Tabela 1 ). As espécies nativas do Cerrado dominaram a paisagem tanto em número de espécies, com (73,2\% do total), quanto em número de indivíduos, (79,5\% do total). Dentre as exóticas, $13,6 \%$ foram espécies brasileiras exóticas ao Cerrado, com 11,6\% dos indivíduos arbóreos e, $15,2 \%$ espécies exóticas estrangeiras com $6,9 \%$ dos indivíduos. O Pequi Caryocar brasiliense foi a espécie nativa mais abundante com $15 \%$ do total de árvores, o Licuri Syagrus picrophylla Barb. Rodr. a mais abundante entre as espécies brasileiras exóticas ao cerrado com $4,6 \%$ e a Palmeira-imperial Roystonea oleracea (Jacq.) O.F. Cook. a exótica estrangeira com $2,9 \%$ do total de árvores. As árvores situadas no local onde foi projetada a canaleta de circulação do BRT, são em sua maioria nativas do Cerrado. Em uma perspectiva conservadora, com a implantação da canaleta projetada de 15 metros de largura no meio do canteiro central, um total de 1.551 indivíduos arbóreos de pelo menos 33 famílias e 96 espécies deverão ser diretamente suprimidos. Destes, 1.371 são árvores nativas do cerrado, 115 provenientes de outras regiões brasileiras e 65 árvores exóticas provenientes de outros países. Das espécies nativas, entre as mais impactadas destacam-se os 232 indivíduos arbóreos de Pequi, 128 Canudos-de-pito, 115 Cachamorras, 113 Cajús Anacardium occidentale, 91 Guaramirins Myrcia fallax, 59 Sucupiras-do-cerrado Bowdichia virgilioides e 45 Favas-de-bolota Parkya platycephala. As árvores são em sua maioria adultas (89\%) e chegam a alcançar os 17 metros de altura; 99,2\% encontram-se em bom estado de desenvolvimento e aproximadamente $80 \%$ apresentam bom estado fitossanitário (Tabela 2). 
TABELA 1 - Famílias, espécies, nome popular, origem, número (N) e frequência (FR) de árvores encontradas no canteiro central da avenida Teotônio Segurado, em Palmas, Tocantins, durante levantamentos realizados em 2016. Quanto a origem as árvores foram classificadas em nativas do cerrado $(\mathrm{N})$, exóticas ao cerrado $(\mathrm{EC})$ e exóticas estrangeiras (EB).

\begin{tabular}{|c|c|c|c|c|c|}
\hline \multirow{2}{*}{ Família } & \multirow{2}{*}{ Espécies } & \multirow{2}{*}{ Nome Popular } & \multirow{2}{*}{ Origem } & \multicolumn{2}{|c|}{ Total } \\
\hline & & & & $\mathbf{N}$ & FR \\
\hline \multirow{5}{*}{ Anacardiaceae } & Anacardium microcarpum Ducke & Cajuí & $\mathrm{N}$ & 25 & 0,006 \\
\hline & Anacardium occidentale L. & Caju & $\mathrm{N}$ & 173 & 0,044 \\
\hline & Mangifera indica $L$. & Manga & EB & 35 & 0,009 \\
\hline & Myracrodruon urundeuva Allemão & Aroeira & $\mathrm{N}$ & 3 & 0,001 \\
\hline & Spondias mombin $L$. & Cajá-mirim & $\mathrm{N}$ & 1 & 0,000 \\
\hline \multirow{4}{*}{ Annonaceae } & Annona classiflora Mart. & Araticum & $\mathrm{N}$ & 16 & 0,004 \\
\hline & Annona coriacea Mart. & Marolo & $\mathrm{N}$ & 2 & 0,001 \\
\hline & Annona glabra $L$. & Araticum-do-brejo & $\mathrm{N}$ & 1 & 0,000 \\
\hline & Xylopia aromatica (Lam.) Mart. & Pimenta-de-macaco & $\mathrm{N}$ & 12 & 0,003 \\
\hline \multirow{2}{*}{ Apocynaceae } & Aspidosperma macrocarpon Mart. & Pequiá & $\mathrm{N}$ & 6 & 0,002 \\
\hline & Hancornia speciosa Gomes & Mangaba & $\mathrm{N}$ & 11 & 0,003 \\
\hline \multirow[t]{6}{*}{ Araliaceae } & Didymopanax morototonii (Aubl.) Decne \& Planch. & Mandioqueiro & $\mathrm{N}$ & 5 & 0,001 \\
\hline & Acrocomia aculeata (Jacq.) Lodd. ex Mart. 1845 & Macaúba & $\mathrm{N}$ & 9 & 0,002 \\
\hline & Attalea phalerata Mart. ex Spreng. & Bacuri & $\mathrm{N}$ & 1 & 0,000 \\
\hline & Caryota urens $L$. & $\begin{array}{c}\text { Palmeira-rabo-de- } \\
\text {-peixe }\end{array}$ & EB & 3 & 0,001 \\
\hline & Cocos nucifera $L$. & Coco-da-bahia & $\mathrm{EC}$ & 56 & 0,014 \\
\hline & Oenocarpus bacaba Mart. & Bacaba & $\mathrm{N}$ & 2 & 0,001 \\
\hline \multirow[t]{6}{*}{ Arecaceae } & Phoenix roebelenii O'Brien & Palmeira-fênix & EB & 7 & 0,002 \\
\hline & Roystonea oleracea (Jacq.) O.F. Cook. & Palmeira-imperial & EB & 116 & 0,029 \\
\hline & Syagrus cocoides Mart. & Pati & $\mathrm{N}$ & 1 & 0,000 \\
\hline & Syagrus oleracea (Mart.) Becc. & Gueroba & $\mathrm{N}$ & 3 & 0,001 \\
\hline & Syagrus picrophylla Barb.Rodr. & Licuri & $\mathrm{EC}$ & 182 & 0,046 \\
\hline & Syagrus romanzoffiana (Cham.) Glassman & Jerivá & $\mathrm{N}$ & 85 & 0,021 \\
\hline
\end{tabular}




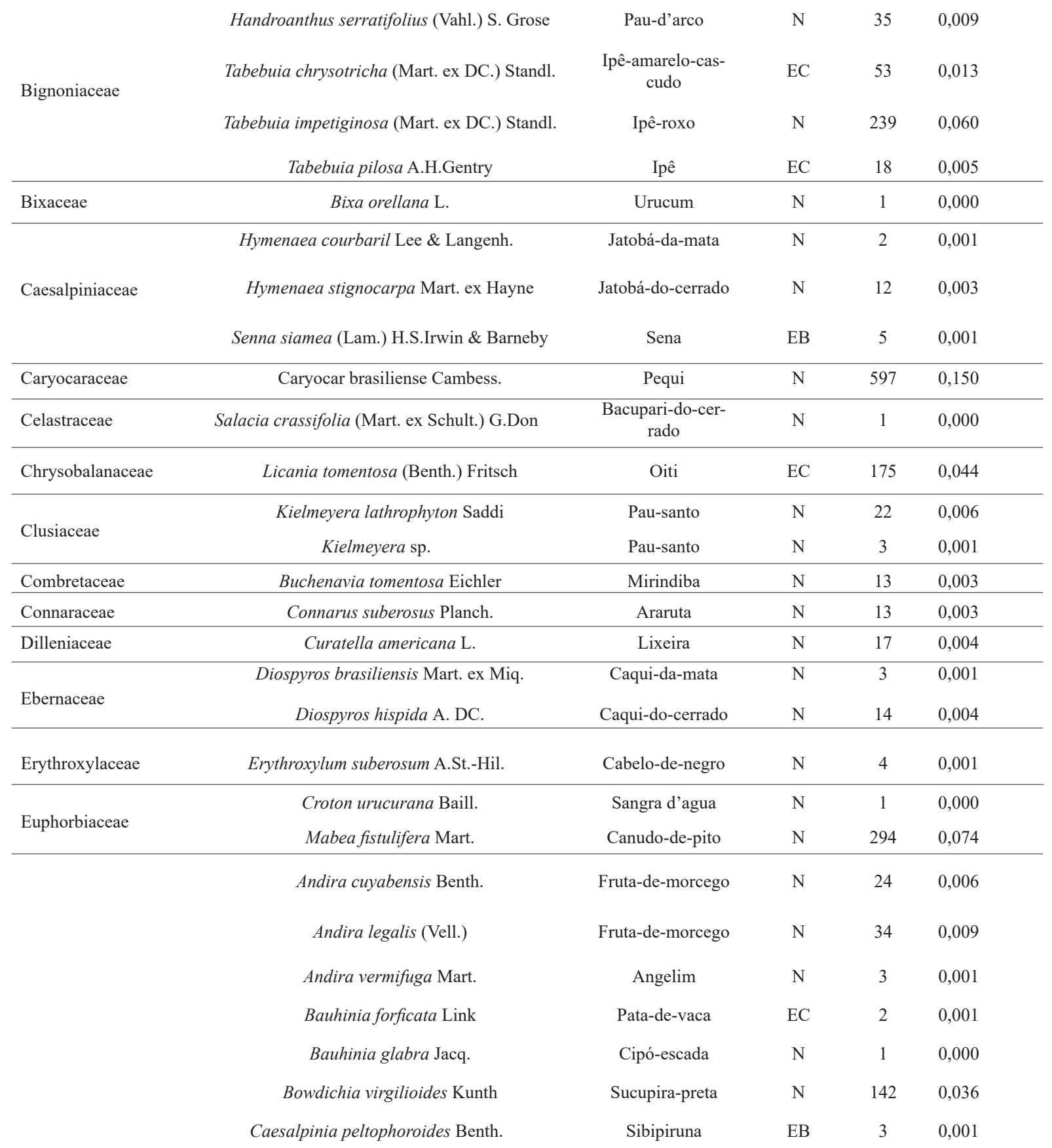


Calliandra brevipes Benth.

Copaifera langsdorffii Desf.

Fabaceae

Delonix regia (Bojer ex Hook.) Raf.
Dimorphandra mollis Benth.

Enterolobium contortisiliquum (Vell.) Morong.

Inga edulis Mart.

Leucaena leucocephala (Lam.) de Wit

Libdibia ferrea (Mart. ex Tul.) L.P.Queiroz

Machaerium acutifolium Vogel

Parkia platycephala Benth.

Pterodon emarginatus Vogel

Pterodon polygalaeflorus Benth.

Tachigali aurea Tul.

Tachigali rubiginosa (Mart. ex Tul.) Oliveira-Filho

Tamarindus indica L.

Vatairea macrocarpa (Benth.) Ducke

\begin{tabular}{|c|c|}
\hline Lamiaceae & Aegiphila lhotskiana cham. \\
\hline \multirow{2}{*}{ Lythraceae } & Lafoensia pacari A.St.-Hil. \\
\hline & Physocalymma scaberrimum Pol \\
\hline \multirow{5}{*}{ Malpighiaceae } & Byrsonima pachyphylla A. Ju \\
\hline & Byrsonima sericea DC. \\
\hline & Heteropterys byrsonimifolia $\mathrm{A}$. Ju \\
\hline & Lophantera lactaescens Juss \\
\hline & Malpighia emarginata D.C. \\
\hline
\end{tabular}

Apeiba tibourbou Aubl.

Malvaceae

Eriotheca gracilipes (K. Schum) A. Robyns

Esponjinha
Copaíba

EC

$\mathrm{N}$

Jacarandá-do-cerrado

Flamboyant

Falso-barbatimão

Tamboril

Ingá-de-metro

Leucena

Pau-ferro

Jacarandá-bico-de-papagaio

Fava-de-bolota

Sucupira-do-cerrado

Sucupira-lisa

$$
\text { Carvoeiro }
$$

Cachamorra

Tamarindo

Amargoso

Tamanqueiro

Dedaleiro

Cega-machado

Murici-ferrugem

\section{Murici}

Murici-macho

Lanterneiro

Acerola

Escova-de-macaco

Paineira-lisa

N

$50 \quad 0,013$ 
Eriotheca pubescens (Mart. \& Zucc.) Schott \& Endl.

Luehea sp.

Sterculia striata A.St.-Hil. \& Naudin

Bellucia grossularioides (L.) Triana

Melastomataceae

\begin{tabular}{c} 
Mouriri pusa Gardner \\
\hline Cedrela fissilis Vell. \\
\hline Mimosa caesalpiniifolia Benth.
\end{tabular}

Mimosaceae

Plathymenia reticulata Benth.

Stryphnodendron adstringens (Mart.) Coville

Artocarpus heterophyllus Lam.

Moraceae

Brosimum gaudichaudii Trécul

Ficus benjamina L.

Eugenia uniflora L.

Myrcia fallax (Rich.) DC.

Psidium guajava L.

Myrtaceae

Psidium myrsinites D.C.

Syzygium cumini (L.)

Syzygium jambos (L.)

Syzygium malaccense (L.) Merr. \& L. M. Perry

\begin{tabular}{lll}
\hline Nyctaginaceae Bougainvillea glabra Choisy \\
\hline
\end{tabular}

Rubiaceae

Ferdinandusa elliptica (Pohl) Pohl

Rutaceae

Sapindaceae

Sapindus saponaria L.

\begin{tabular}{lccccc}
\hline Sapotaceae & Pouteria ramiflora (Mart.) Radlk. & Massaranduba & N & 13 & 0,003 \\
\hline Simaroubaceae & Simarouba versicolor A.St.-Hil. & Mata-menino & N & 30 & 0,008 \\
\hline Sterculiaceae & Guazuma ulmiofolia Lam. & Mutamba & N & 4 & 0,001 \\
\hline
\end{tabular}




\begin{tabular}{|c|c|c|c|c|c|}
\hline Urticaceae & Cecropia pachystachya Trécul. & Embaúba & $\mathrm{N}$ & 36 & 0,009 \\
\hline \multirow{2}{*}{ Vochysiaceae } & Qualea grandiflora Mart. & $\begin{array}{c}\text { Pau-terra-folha- } \\
\text {-grande }\end{array}$ & $\mathrm{N}$ & 67 & 0,017 \\
\hline & Salvertia convallariodora A.St.-Hil. & Chapéu-de-couro & $\mathrm{N}$ & 24 & 0,006 \\
\hline
\end{tabular}

TABELA 2 - Elementos removidos e grupos taxonômicos afetados na arborização do canteiro central da Avenida Teotônio Segurado com a implantação projetada $(15 \mathrm{~m})$ e alternativa $(7,5 \mathrm{~m})$ proposta para as canaletas de circulação do BRT em Palmas, Tocantins.

\begin{tabular}{|c|c|c|}
\hline Impacto & Canaleta central com $15 \mathrm{~m}$ & $\begin{array}{c}\text { Canaletas laterais com } \\
7,5 \mathrm{~m}\end{array}$ \\
\hline Indivíduos arbóreos & 1.551 & 1.095 \\
\hline Famílias & 33 & 29 \\
\hline Espécies & 96 & 73 \\
\hline Árvores Nativas & 1.371 & 623 \\
\hline Árvores exóticas brasileiras & 115 & 293 \\
\hline Árvores exóticas estrangeiras & 65 & 136 \\
\hline Árvores Adultas & 1.380 & 842 \\
\hline Árvores em bom estado de desenvolvimento & 1.539 & 1.091 \\
\hline Árvores em bom estado fitossanitário & 1.226 & 861 \\
\hline
\end{tabular}

Com a implantação de canaletas projetadas com 7,5 metros de largura, situadas nas extremidades do canteiro central da avenida, estima-se que aproximadamente 1.095 indivíduos arbóreos de pelo menos 29 famílias e 73 espécies deverão ser diretamente suprimidos. Destes, 623 são nativos do cerrado, 293 provenientes de outras regiões brasileiras e 136 árvores exóticas provenientes de outros países. Neste traçado, as espécies mais impactadas seriam as Palmeiras (Arecaceae) sendo 181 Licuris, 107 Palmeiras-imperiais e 77 Jerivás Syagrus romanzoffiana. Dentre as árvores nativas 107 são Pequis, 46 Amargosos Vatairea macrocarpa e 45 Canudos-de-pito. Neste trecho, a grande maioria das árvores e palmeiras também é adulta $(76,9 \%)$ com altura máxima de 16 metros. Destas, $99 \%$ estão em bom estado de desenvolvimento e $79 \%$ em bom estado fitossanitário (Tabela 2). 


\section{Discussão}

As espécies arbóreas, no que lhes concerne, dominam a paisagem de grande parte dos canteiros da avenida Teotônio Segurado. Entretanto, esta representatividade é bem menor quando consideramos apenas os canteiros que receberam tratamento paisagístico, já que as palmeiras são bastante frequentes nestes trechos e os arbustos raramente utilizados. Ainda que existam grandes espaços vazios, o incremento da arborização no canteiro central da Av. Teotônio Segurado tem sido pouco frequente nos últimos anos.

A grande maioria das árvores analisadas encontra-se em bom estado fitossanitário, porém alguns indivíduos necessitam de uma avaliação individual. Entre as espécies exóticas, a Palmeira-imperial Roystonea oleracea e a Palmeira-rabo-de-peixe Caryota urens foram aquelas com maior proporção de indivíduos com problemas. Entre as nativas do cerrado, a maioria dos problemas fitossanitários esteve relacionada à presença de cupins, formigas e/ou fungos, sendo a Cachamorra Tachigali rubiginosa, o Falso-barbatimão Dimorphandra mollis, o Jerivá Syagrus romanzoffiana, a Painera-peluda Eriotheca pubescens e o Guraramirim Myrcia fallax as espécies mais afetadas.

Já as espécies nativas autóctones, situadas nos trechos de canteiros, que não sofreram intervenção paisagística, formam grandes bosques lineares que se destacam pela diversidade e composição das espécies. Árvores de grande porte como o Jatobá-do-cerrado Hymenaea stigonocarpa, Sucupira-do-cerrado Bowdchia virgilioides, Sucupira-lisa Pterodron emarginatus e o Vinhático Platimenia reticulata, são numerosas no canteiro central e possuem um alto valor econômico, ecológico e conservacionista, devendo ser preservadas.

Somam-se a essas, espécies frutíferas como o Pequi, a Mirindiba Buchenavia tomentosa, os diversos Muricis Byrsonima sp., Mangaba Hancornia speciosa, Macaúba Acromia aculeata, Jatobás Hymenaea sp., Cajui Anacardium microcarpum, entre outras frutíferas do cerrado, que são consideradas imunes ao corte e devem ser protegidas segundo a Constituição do Estado do Tocantins, Art. 112 e pelo Decreto No 838, de 13 de outubro de 1999.

É evidente que a implantação da canaleta de circulação do BRT no meio do canteiro central da Av. Teotônio Segurado, ainda que avaliada de maneira conservadora, causará um impacto significativamente maior à arborização local. Em números absolutos haverá uma perda de pelo menos 456 indivíduos arbóreos a mais quando comparada com as canaletas laterais: um impacto aproximadamente $30 \%$ maior. Em termos de composição, importância biológica e ecológica, haverá uma perda considerável de biodiversidade e funcionalidade devido à retirada de muitas espécies autóctones, ou seja, nascidas naturalmente no local e o impacto com a supressão de pelo menos 23 espécies nativas do Cerrado a mais do que a implantação das canaletas nas laterais da avenida. Deve-se considerar ainda que muitas dessas espécies estão protegidas pela Legislação Estadual e têm um papel fundamental para a qualidade dos serviços ambientais e ecossistêmicos na área urbana de Palmas.

Diversos estudos têm demonstrado que as árvores são responsáveis por múltiplos benefícios socioambientais e econômicos, melhorando a qualidade do ar e o microclima, fornecendo sombra, reduzindo a poluição sonora e do ar, o estresse e o consumo de energia, entre outros fatores extre- 
mamente relevantes para o meio urbano (Abreu \& Labaki, 2010; Soares et al., 2011; Mullaney et al., 2015; McPherson et al., 2016). Neste contexto, a arborização do canteiro central da avenida Teotônio Segurado, por suas diversas características, desempenha um papel fundamental para a melhoria da qualidade de vida na cidade, proporcionando a ela benefícios ambientais, sociais e econômicos. No entanto, muitas destas vantagens não se enquadram em apenas uma categoria e em última instância, benefícios ambientais também são benefícios sociais ou econômicos e vice-versa.

Nos últimos 30 anos, observa-se uma mudança nos propósitos de implantação da arborização urbana, passando de um padrão estético ornamental para um contexto mais amplo que inclui a provisão de serviços ambientais relacionados à qualidade do ar, conservação de energia ou redução do impacto de águas pluviais (Seamans, 2013). Apesar de ser uma cidade planejada e com poucos anos, Palmas já apresenta problemas advindos do processo de urbanização que poderiam ser amenizados com uma boa gestão da arborização. Além disso, o paisagismo urbano local tem-se baseado, desde a sua criação, em critérios majoritariamente estéticos.

Um canteiro central como o da Av. Teotônio Segurado, amplo e com considerável adensamento arbóreo em alguns trechos, exerce um papel importante no conforto térmico local. A cidade, com seus 28 anos, já apresenta ilhas de calor nas regiões com maior adensamento humano e baixa cobertura arbórea, cuja temperatura pode elevar-se a mais de $7^{\circ} \mathrm{C}$ com respeito à temperatura oficial ou até mais de $10^{\circ} \mathrm{C}$ com respeito às áreas mais arborizadas da cidade (Paz, 2009). Estudos semelhantes têm sido realizados no Brasil (Shams et al., 2009; Tejas et al., 2011) e em outros países (Zhang et al., 2007;
Yilmaz et al., 2008) mostrando a relação positiva da arborização com a melhoria do conforto térmico urbano. Ademais, nas ruas e avenidas de cidades tropicais com déficit de arborização, o incremento da temperatura na superfície do pavimento aumenta a sensação de desconforto térmico para pedestres, inibido o uso de transportes públicos ou alternativos, como a bicicleta, além de estimular motoristas a fazerem uso do ar-condicionado veicular (Vailshery et al., 2013).

Porém, o efeito de resfriamento promovido pela arborização está diretamente relacionado ao tamanho da árvore e de sua copa, sua localização e densidade (Mullaney et al., 2015). A eficácia dos bosques urbanos sobre o clima local é proporcional ao percentual de área de bosque sobre o total urbanizado (Dacanal, 2011). Considerando o fato de Palmas ser uma das capitais mais quentes do país, é imperativo que se promova o incremento e maior adensamento da arborização no seu canteiro central, e não a sua supressão, ainda que parcial, como planejado. Estima-se de cinco a dez anos para que as árvores plantadas possam atingir um porte e altura suficientes para influenciar positivamente o microclima local. Considerando que aproximadamente $80 \%$ do efeito de resfriamento originado pelas árvores resulta diretamente do sombreamento (Shashua-Bar et al., 2009), a remoção de árvores adultas, de médio e grande porte, que em alguns trechos formam dosséis contínuos, efetivamente exercendo uma função bioclimática, e sua substituição por árvores jovens causaria um impacto significativo no conforto térmico e na qualidade do ar local, acrescido do fluxo de ônibus do BRT.

A manutenção da arborização na avenida Teotônio Segurado tem sua importância elevada por ela estar situada ao longo da principal via de 
tráfego da cidade. E, considerando que as emissões de gases poluentes e o ruído emitido pelo tráfego de veículos estão entre os principais problemas de saúde pública no meio urbano (Toledo \& Nardocci, 2011), as árvores funcionariam como barreira sonora, reduzindo a propagação do som e capturando diversos poluentes como ozônio, óxidos de nitrogênio, óxido e dióxido de enxofre, monóxido e dióxido de carbono e material microparticulado (Tallis \& Polasky, 2011; Vailshery et al., 2013). Novamente, os indivíduos arbóreos maiores são mais eficientes, removendo de 60 a 70 vezes mais poluentes do que os menores (McPherson et al., 1994). A arborização viária, em especial aquela situada entre as vias de rodagem, além de criar um ambiente mais agradável pelo sombreamento e ter elevado valor estético, servem de referência, definindo os limites da via, auxiliando motoristas e tornando o trânsito mais seguro (Tarran, 2009).

Outro aspecto relevante da arborização da avenida, diz respeito ao seu papel na interceptação da chuva e sua infiltração no solo. A compactação e impermeabilização do solo urbano e a intensidade das chuvas na região entre os meses de novembro a março auxiliam na formação de enxurradas que comprometem a circulação no principal eixo rodoviário de Palmas, gerando inúmeros transtornos e colocando em risco a população. Pesquisas mostram que o escoamento das águas pluviais é maior em superfícies impermeabilizadas quando comparado com solos arborizados e, dependendo das características locais e espécies arbóreas, pode resultar em uma economia substancial nos custos relacionados à infraestrutura de drenagem urbana (McPherson et al., 1999; McPherson et al., 2005; McPherson et al., 2011). O canteiro central, por sua dimensão, localização e densidade arbórea em alguns trechos, poderia funcionar como uma grande caixa de contenção da água pluvial que flui no sentido leste/ oeste. Para otimizar sua eficácia seriam necessárias pequenas intervenções estruturais e aumentar o adensamento arbóreo nos trechos de canteiro com déficit de arborização.

As árvores no meio urbano também fornecem habitat para a fauna, existindo uma associação entre a riqueza dessa e a abundância e composição das espécies arbóreas (Alvey, 2006; Brun et al., 2007). A manutenção de indivíduos maduros e o adensamento arbóreo têm sido reportados como fatores fundamentais para a riqueza e abundância de aves no meio urbano (Barth et al., 2015). Trabalhos realizados em Palmas por Reis et al. (2012) demonstraram haver uma correlação positiva entre a riqueza de aves do cerrado e a densidade e a estrutura da vegetação nativa, enquanto que a arborização exótica exerce um efeito negativo, reduzindo tal riqueza. Além disso, os autores observaram uma maior riqueza e abundância de aves em áreas urbanizadas com vegetação nativa contínua do que nas áreas semi ou integralmente urbanizadas.

A expansão urbana acelerada promove a perda e fragmentação dos habitats naturais, causando perturbações significativas na fauna silvestre. Nesse sentido, a manutenção de fragmentos de habitat remanescente, como áreas verdes, praças e parques, e a sua conexão por meio de corredores ecológicos através da matriz urbana tem o potencial de incrementar a diversidade biológica nas cidades (Barth et al., 2015). A presença de ambientes de relevada importância para a biodiversidade no meio urbano, por sua vez, nos fornece benefícios ambientais e econômicos, considerando que nesses ambientes predominam as trocas genéticas entre espécies vegetais e a promoção da biodiversidade da fauna e flora, 
e é no meio urbano que exercem um papel ainda mais considerável: funcionam como verdadeiros filtros ambientais, reduzindo a poluição atmosférica, o ruído, a temperatura ou fornecem áreas de recreação. Tais ambientes remanescentes devem, então, integrar a estrutura ecológica urbana para maximizar sua eficiência (Pauleit et al., 2011).

Outra importância fundamental do canteiro central desta avenida para a estrutura ecológica urbana da cidade se mostra quando temos em mente que ele conecta diversas áreas de proteção permanente da área urbana, regidas pelo Código Florestal (Brasil, 2012) e/ou áreas protegidas como as Unidades de Conservação estabelecidas pelo Plano Diretor de Palmas (LC 155/2007). Estas áreas protegidas estão em sua maioria ao longo dos cursos d'água dos ribeirões que nascem ou cortam a cidade no sentido leste/oeste (Coriolano \& Pinheiro, 2011). Além de proteger as nascentes e os recursos hídricos, elas protegem as matas de galeria, uma das fitofisionomias do cerrado mais relevantes em termos de biodiversidade, pois abrigam a maior diversidade de vertebrados desse bioma (Johnson $e t$ al., 1999). Na cidade em foco, as matas de galeria também são responsáveis por abrigar grande parte das 346 espécies de aves encontradas em sua área urbana e entorno (Pinheiro et al., 2008; Barbosa et al., 2015).

O Plano de Arborização Urbana de Palmas (Palmas, 2016), ressalta a importância dos corredores verdes e da estrutura ecológica urbana como instrumento de ordenamento e planejamento territorial na melhoria da qualidade de vida no meio urbano, destacando a importância da avenida Teotônio Segurado como um grande corredor verde conectando as áreas protegidas urbanas e formando um grande parque linear, integrado ao planejamento regional de conservação da biodiversidade. No meio urbano, os parques são considerados um dos caminhos mais efetivos de promoção do bem-estar com benefícios diretos para a saúde humana e melhorando a qualidade do ambiente local.

\section{Considerações finais}

Ainda que o planejamento da cidade, descrito no Plano Básico/Memória (Grupo Quatro, 1989), tenha previsto que o canteiro central da avenida Teotônio Segurado fosse uma área verde, mas que no futuro, devido a possíveis ajustes tecnológicos, pudesse conter pistas exclusivas de ônibus ou até mesmo um metrô a céu aberto, ele não previu o impacto ambiental que isso representaria para a cidade 28 anos depois, uma vez que a implantação suprimiu extensas áreas verdes naturais, onde o crescimento urbano vem reduzindo significativamente a cobertura vegetal, com consequente formação de ilhas de calor em uma capital cuja taxa de crescimento é a maior do país

O traçado original das canaletas de circulação do sistema de transporte BRT sobre a parte central do canteiro da avenida Teotônio Segurado terá um impacto aproximadamente $30 \%$ maior sobre a arborização, quando comparado à alternativa de implantação das canaletas em suas extremidades, suprimindo cerca de 456 indivíduos arbóreos a mais do que a segunda alternativa de traçado.

Já a manutenção e o aumento da arborização do canteiro da avenida, como anteriormente aqui se expusera, proporcionarão inúmeros benefícios sociais, ambientais e econômicos para a cidade, dado que este funcionará como um grande corredor ecológico (parque linear), interligando as áreas 
verdes urbanas (parques, jardins, praças, etc.), áreas de preservação permanente e unidades de conservação, promovendo a manutenção da biodiversidade urbana, além de funcionar como um grande filtro ambiental, reduzindo a poluição atmosférica e sonora, influenciando no conforto térmico, reduzindo as ilhas de calor, e fornecendo opção para implantação de áreas de recreação.

A implantação das canaletas de circulação do BRT nas laterais da avenida, por outro lado, é ambientalmente e socioeconomicamente a alternativa mais viável para a atualidade. Esta alternativa implicará em um menor impacto sobre a arborização do canteiro, promovendo a manutenção de um maior número de indivíduos de espécies nativas do cerrado. É, portanto, necessário refletir sobre os custos e benefícios de implantação do sistema de transporte coletivo BRT sobre o canteiro central da avenida Teotônio Segurado. Esse sistema, no que lhe diz respeito, apresenta diversas vantagens socioeconômicas e ambientais quando comparadas ao sistema de transporte urbano tradicional, e não tão evidentes quando comparadas com outros sistemas modais de transporte urbano (Branco, 2013). No entanto, a grande maioria dos projetos tem sido implantada em cidades com grande contingente populacional, onde as opções de implantação das vias são limitadas, uma realidade bem diferente da encontrada em Palmas.

\section{Agradecimentos}

Agradecemos o apoio da Fundação de Apoio Científico e Tecnológico do Tocantins - FAPTO, a Fundação Municipal de Meio Ambiente de Palmas-TO e Lojas Havan.

\section{Referências}

Abreu, L. V.; Labaki, L. C. Conforto térmico propiciado por algumas espécies arbóreas: avaliação do raio de influência através de diferentes índices de conforto. Ambiente Construido, 10(4), 103-117, 2010. Disponível em: http:// www.seer.ufrgs.br/index.php/ambienteconstruido/article/ view/12359/10607

Alves, H. P. F. Metodologias de interação de dados sociodemográficos e ambientais para análise da vulnerabilidade socioambiental em áreas urbanas no contexto das mudanças climáticas. In: Hogan, D.; Marandola Jr., E. (Orgs.). População e mudança climática: dimensões humanas das mudanças ambientais globais. Brasília: UNFPA, 2009, p. 75-105.

Alvey, A. A. Promoting and preserving biodiversity in the urban forest. Urban Forestry \& Urban Greening, 5, p. 195201, 2006. doi: 10.1016/j.ufug.2006.09.003

Barbosa, M. de O.; Dornas, T.; Dionísio, C.; Marcelino, D. G. Novos registros ornitológicos para a região de Palmas, Tocantins. Atualidades Ornitológicas, 188, 59-65, 2015. Disponível em: http://www.ao.com.br/download/ AO188_59.pdf

Barth, B. J.; Ian, S.; Wilson, R. S. New urban developments that retain more remnant trees have greater bird diversity. Landscape and Urban Planning 136, 122-129, 2015. doi: org/10.1016/j.landurbplan.2014.11.003

Benedict, M. A.; McMahon, E. T. Green Infrastructure: Linking Landscapes and Communities. Washington: Island Press, 2006.

Branco, S. P. V. M. Estudo e aplicação de sistemas BRT - Bus Rapid Transit. Porto Dissertação (Mestrado em Engenharia Civil), Universidade do Porto, 2013.

Brasil. Lei no ${ }^{\circ} 12.651$ de 25 de maio de 2012. Dispõe sobre a proteção da vegetação nativa; altera as Leis nos 6.938, de 31 de agosto de 1981, 9.393, de 19 de dezembro de 1996, e 11.428, de 22 de dezembro de 2006; revoga as Leis nos 4.771, de 15 de setembro de 1965, e 7.754, de 14 de abril de 1989, e a Medida Provisória no 2.166-67, de 24 de agosto de 2001; e dá outras providências. Brasília: DOU de $28 / 05 / 2012$. 
Brun, F. G. K.; Link, D.; Brun, E. J. O emprego da arborização na manutenção da biodiversidade da fauna em áreas urbanas. REVSBAU, 2(1), 117-127, 2007. Disponível em: http://www.revsbau.esalq.usp.br/artigos_revisao/revisao01. pdf

Campos, V. B. G. Planejamento de transportes: conceitos e métodos. Editora Interciência, 2013.

Carvalhêdo, W. S.; Lira, E. R. Palmas ontem e hoje: do interior do cerrado ao Portal da Amazônia. Observatorium: Revista Eletrônica de Geografia, 1(2), 51-73, 2009. Disponível em: http://www.observatorium.ig.ufu.br/pdfs/1edicao/ n2/PALMAS\%20ONTEM\%20E\%20HOJE.pdf

Chaves, S. T. Estudo de caso - a cidade de Juiz de Fora MG - sua centralidade e problemas socioeconômicos. Revista GEOMAE, 2(1), 155-170, 2011. Disponível em: http://www.fecilcam.br/revista/index.php/geomae/article/ viewFile/150/pdf_33

Coriolano, C. P.; Pinheiro, R. T. Unidades de conservação em área urbana. O (des)caso de Palmas/TO. Revista Jus Navigandi, 3002, 2011. Disponível em: https://jus.com.br/ artigos/20025. Acesso em: out. 2017.

Dacanal, C. Fragmentos florestais urbanos e interações climáticas em diferentes escalas: estudos em Campinas, SP. Campinas, Tese (Doutorado em Arquitetura e Urbanismo), Universidade Estadual de Campinas, 2011.

Ferreira, J. C.; Silva, C.; Tenedorio, J. A.; Pontes, S.; Encarnação, S.; Marques, L. Coastal Greenways: Interdisciplinarity and Integration Challenges for the Management of Developed Coastal Areas. Journal of Coastal Research, 2004.

Ferreira, J. C. Estrutura ecológica e corredores verdes - estratégias territoriais para um futuro urbano sustentável. In: $4^{\circ}$ Congresso Luso Brasileiro para Planejamento Urbano Regional, Integrado Sustentável. Faro, 6 out., 2010.

Flora do Brasil. Jardim Botânico do Rio de Janeiro. Disponível em: < http://floradobrasil.jbrj.gov.br/ > . Acesso em: mai. 2017

Forest Research. Benefits of green infrastructure. Report by Forest Research. Forest Research, Farnham, 2010.

Grupo Quatro S/C. Projeto da capital do estado do Tocan- tins: plano básico/memória. Palmas: Governo do Estado do Tocantins, 1989.

Hogan, D. J.; Cunha, J. M. P.; Carmo, R. L.; Oliveira, A. A. B. Urbanização e vulnerabilidade socioambiental: o caso de Campinas. In: Hogan, D. J.; Baeninger R.; Cunha, J. M. P. da; Carmo, R. Luiz do. (Org.). Migração e ambiente nas aglomerações urbanas. Campinas: Nepo/Unicamp, p. 395-418, 2001.

IBGE - Instituto Brasileiro de Geografia e Estatística. IBGE divulga as estimativas populacionais dos municípios, 2017. Disponível em: $<$ https://agenciadenoticias.ibge.gov. br/agencia-sala-de-imprensa/2013-agencia-de-noticias/ releases/16131-ibge-divulga-as-estimativas-populacionais-dos-municipios-para-2017.html>. Acesso em: out. 2017.

Johson, M. A.; Saraiva, P. M.; Coelho, D. The role of gallery forest in the distribution of Cerrado mammals. Revista de Biologia, 59(3), 421-427, 1999. Disponível em: http://www. scielo.br/pdf/rbbio/v59n3/v59n3a05.pdf

Lorenzi, H. Árvores brasileiras: Manual de identificação e cultivo de plantas arbóreas nativas do Brasil. Nova Odessa, 2002.

Lorenzi, H. Árvores brasileiras: Manual de identificação e cultivo de plantas arbóreas nativas do Brasil. Nova Odessa, 2013.

Lorenzi, H. Árvores brasileiras: Manual de identificação e cultivo de plantas arbóreas nativas do Brasil. Nova Odessa, 2016.

McPherson, E.; Nowak, D. J.; Rowntree, R. A. Chicago's urban forest: results of the Chicago Urban Forest Climate Project. Department of Agriculture, Forest Service, Radnor, 1994.

McPherson, F. G.; Simpson, J. R.; Peper, P. J.; Xiao, Q. Benefit-cost analysis of Modesto's municipal urban forest. Journal of Arboriculture, 25, 235-248, 1999.

McPherson, E. G.; Simpson, J. R.; Peper, P. J.; Maco, S. E.; Xiao, Q. Municipal forest benefits and costs in five U.S cities. Journal of Forestry, 103, 411-416, 2005.

McPherson, E. G.; Simpson, J. R.; Xiao, Q.; Wu, C. Million trees Los Angeles canopy cover and benefit assessmet. Landscape Urban Planning, 99, 40-50, 2011. doi: 10.1016/j. 


\section{landurbplan.2010.08.011}

McPherson, E. G.; Van Doorn, N.; Goede. J. de. Structure, function and value of street trees in California, USA. $U r$ ban Forestry \& Urban Greening, 17, 104-115, 2016. doi: 10.1016/j.ufug.2016.03.013

Mullaney, J.; Lucke, T.; Trueman, S. J. A review of benefits and challenges in growing street trees in paved urban environments. Landscape and Urban Planning, 134, 157-166, 2015. doi: 10.1016/j.landurbplan.2014.10.013

Palmas. Prefeitura Municipal de Palmas. Diagnóstico da Arborização Urbana de Palmas-TO. 2015. 352p.

Palmas. Prefeitura Municipal de Palmas. Plano de arborização Urbana de Palmas-TO. 2016. 116p.

Paz, L. H. F. A influência da vegetação sobre o clima urbano de Palmas-TO. Brasília, Dissertação (Mestrado em Arquitetura e Urbanismo). Universidade de Brasília, 2009.

Pauleit, S.; Liu, L.; Ahern, J.; Kazmierczak, A. Multifunctional Green Infrastructure Planning to Promote Ecological Services in the City. In: Niemelä, J.; Breuste, G.; Guntenspergen, N.; McIntyre, T.; Elmqvist, T.; James, P. (Eds.). Urban ecology: patterns, processes, and applications. Oxford University Press, 2011. p. 272-285.

Pinheiro, R. T.; Dornas, T.; Reis, E. dos S.; Barbosa, M. de O.; Rodello, D. Birds of the urban area of Palmas, TO: composition and conservation. Revista Brasileira de Ornitologia, 16( 4), 339-347, 2008. Disponível em: http:// www4.museu-goeldi.br/revistabrornito/revista/index.php/ BJO/article/view/5205/pdf_ 820

Quintas, A. V.; Curado, M. J. The Urban Green Network as a Quality of Life Promoter. In: Breuste, J.; Kozová, M.; Finka, M. (Eds.). European Landscapes in Transformation: Challenges for Landscape Ecology and Management: Proceedings from the European IALE Conference 2009. Comenius University, 2009, p. 288-292.

Reis, E.; López-Iborra, G. M.; Pinheiro, R. T. Changes in bird species richness through different levels of urbanization: Implications for biodiversity conservation and garden design in Central Brazil. Landscape and Urban Planning, 107, 31-42, 2012. doi: 10.1016/j.landurbplan.2012.04.009

Shashua-Bar, L.; Pearlmutter, D.; Erell, E. The cooling ef- ficiency of urban landscape strategies in a hot dry climate. Landscape and Urban Planning, 92, 179-186, 2009. doi: 10.1016/j.landurbplan.2009.04.005

Seamans, G. S. Mainstreaming the environmental benefits of street trees. Urban Forestry and Urban Greening, 12, 2-11, 2013. doi: 10.1016/j.ufug.2012.08.004

Shams, J. C. A.; Giacomeli, D. C.; Sucomine, N. M. Emprego da arborização na melhoria do conformto térmico nos espaços livres públicos. REVSBAU, 4(4), 1-16, 2009. Disponível em: http://www.revsbau.esalq.usp.br/artigos cientificos/artigo 71.pdf

Soares, A. L.; Rego, F. C.; McPherson, E. G.; Simpson, J. R.; Peper, P. J.; Xiao, Q. Benefits and costs of street trees in Lisbon, Portugal. Urban Forestry and Urban Greening, 10, 69-78, 2011. doi: 10.1016/j.ufug.2010.12.001

Tallis, H.; Polasky, S. Assessing multiple ecosystem services: an integrated tool for the real world. In: Kareiva, P.; Tallis H.; Ricketts T. H.; Daily, G. C.; Polasky, S. (Eds). Natural capital: theory and pracice of mapping ecosystem servives. Oxford University Press, 2011. p. 34-52.

Tarran, J. People and trees, providing benefits, overcominig impediments. In: Lawry, D.; Gardner, J.; Bridget, M. (Eds). Proceedings of the 10th National Street Tree Symposium. Adelaide University, 2009. p. 63-82.

Tejas, G. T.; Azevedo, M. G. F. de.; Locatelli, M. A influência de áreas verdes no comportamento higrotérmico e na percepção ambiental do citadino em duas unidades amostrais no município de Porto Velho, Rondônia, Brasil. REVSBAU, 6(4), 15-34, 2011. Disponível em: http://www.revsbau.esalq. usp.br/artigos_cientificos/artigo192-publicacao.pdf

The Plant List. Version 1.1. Published on the Internet, 2013. Disponível em: $<$ http://www.theplantlist.org/>. Acesso em: mai. 2017.

Tocantins - Secretaria do Planejamento e Orçamento. Base de dados cartográficos do Tocantins, 2012. Disponível em: http://seplan.to.gov.br/zoneamento/. Acesso em: jun. 2017.

Toledo, G. I. F. M.; Nardocci, A. C. Poluição veicular e saúde da população: uma revisão sobre o município de São Paulo (SP), Brasil. Revista Brasileira de Epidemiologia, 14(3), 445-454, 2011. Disponível em: http://www.scielo. 


\section{br/pdf/rbepid/v14n3/09.pdf}

Vailshery, L. S.; Jaganmohan, M.; Nagendra, H. Effect of street trees on microclimate and air pollution in a tropical city. Urban Forestry \& Urban Greening, 12(3), 408-415, 2013. doi: 10.1016/j.ufug.2013.03.002
Yilmaz, H.; Toy, S.; Irmak, M. A.; Yilmaz, S.; Bulut, Y. Determination of temperature diferences between asphalt concrete, soil and grass surfaces of the city of Erzurum, Turkey. Atmosfera, 21, 135-146, 2008.

Zhang, W.; Rickettsb, T. H.; Kremenc, C.; Carneyd, K.; Swintona, S. M. Ecosystem services and dis-services to agriculture. Ecological Economics, 64, 253-260, 2007. doi: 10.1016/j.ecolecon.2007.02.024 